\title{
The general nilpotent operator system
}

\author{
J. Dombi - O. Csiszár
}

\begin{abstract}
In this paper we show that a consistent logical system generated by nilpotent operators is not necessarily isomorphic to Łukasiewicz-logic, which means that nilpotent logical systems are wider than we have earlier thought. Using more than one generator functions we examine three naturally derived negations in these systems. It is shown that the coincidence of the three negations leads back to a system which is isomorphic to Łukasiewicz-logic. Consistent nilpotent logical structures with three different negations are also provided.
\end{abstract}

\section{Introduction}

One of the most significant problems of fuzzy set theory is the proper choice of set-theoretic operations $[23,27]$. Triangular norms and conorms have thoroughly been examined in the literature $[16,11,10,13]$. The most well-characterized class of t-norms are the so-called representable t-norms. They are derived from the solution of the associative functional equation [1]. The two main types of representable t-norms are the strict and non-strict or nilpotent t-norms.

t-norms and t-conorms are often used as conjunctions and disjunctions in logical structures [12], [19]. Eukasiewicz fuzzy logic $[14,18,20,21]$ is the logic where the conjunction is the Eukasiewicz tnorm. It has been introduced for philosophical reasons by Lukasiewicz in [18] and it is among the most significant and widely examined non-classical logics.

The class of non-strict t-norms has preferable properties which make them more usable in building up logical structures. Among these properties are the fulfillment of the law of contradiction and the excluded middle, the continuity of the implication or the coincidence of the residual and the Simplication $[8,26]$. Due to the fact that all continuous Archimedean (i.e. representable) nilpotent t-norms are isomorphic to the Łukasiewicz t-norm [13], the previously studied nilpotent systems were all isomorphic to the well-known Łukasiewicz-logic.

In this paper we show that a logical system generated by nilpotent operators is not necessarily isomorphic to Łukasiewicz-logic. Of course, this lack of isomorphy is not the result of introducing a new operator family, it simply means that the system itself is built up in a significantly different way using more than one generator functions.

The paper is organized as follows. After some preliminaries in Section 2 we give a characterization of negation operators in Section 3, as negations will have an important role to play in Section 4. After considering the class of connective systems generated by nilpotent operators, we examine their structural properties in Section 4. We show examples for bounded systems, i.e. consistent nilpotent systems which are not isomorphic to Łukasiewicz-logic. Necessary and sufficient conditions are given for these systems to satisfy the De Morgan law, classification property and consistency. A wide range of examples for consistent and non-consistent bounded systems can be found in Section 5 . 


\section{Preliminaries - basic fuzzy connectives}

\section{$2.1 \quad$ t-norms and t-conorms}

First, we recall some basic notations and results regarding t-norms, t-conorms and negation operators that will be useful in the sequel.

A triangular norm (t-norm for short) $T$ is a binary operation on the closed unit interval $[0,1]$ such that $([0,1], T)$ is an abelian semigroup with neutral element 1 which is totally ordered, i.e., for all $x_{1}$, $x_{2}, y_{1}, y_{2} \in[0,1]$ with $x_{1} \leq x_{2}$ and $y_{1} \leq y_{2}$ we have $T\left(x_{1}, y_{1}\right) \leq T\left(x_{2}, y_{2}\right)$, where $\leq$ is the natural order on $[0,1]$.

A triangular conorm ( $t$-conorm for short) $S$ is a binary operation on the closed unit interval $[0,1]$ such that $([0,1], S)$ is an abelian semigroup with neutral element 0 which is totally ordered.

Standard examples $[5,16]$ of t-norms are the minimum $T_{\mathbf{M}}$, the product $T_{\mathbf{P}}$, the Eukasiewicz t-norm $T_{\mathbf{L}}$ given by $T_{\mathbf{L}}(x, y)=\max (x+y-1,0)$, and the drastic product $T_{\mathbf{D}}$ with $T_{\mathbf{D}}(1, x)=T_{\mathbf{D}}(x, 1)=x$, and $T_{\mathbf{D}}(x, y)=0$ otherwise.

Standard examples of t-conorms are the maximum $S_{\mathbf{M}}$, the probabilistic sum $S_{\mathbf{P}}$, the Łukasiewicz t-conorm $S_{\mathbf{L}}$ given by $S_{\mathbf{L}}(x, y)=\min (x+y, 1)$, and the drastic $\operatorname{sum} S_{\mathbf{D}}$ with $S_{\mathbf{D}}(0, x)=S_{\mathbf{D}}(x, 0)=x$, and $S_{\mathbf{D}}(x, y)=1$ otherwise.

A continuous t-norm $\mathrm{T}$ is said to be Archimedean if $T(x, x)<x$ holds for all $x \in(0,1)$, strict if $T$ is strictly monotone i.e. $T(x, y)<T(x, z)$ whenever $x \in(0,1]$ and $y<z$, and nilpotent if there exist $x, y \in(0,1)$ such that $T(x, y)=0$.

From the duality between t-norms and t-conorms we can easily get the following properties as well. A continuous t-conorm $\mathrm{S}$ is said to be Archimedean if $S(x, x)>x$ holds for every $x, y \in(0,1)$, strict if $S$ is strictly monotone i.e. $S(x, y)<S(x, z)$ whenever $x \in[0,1)$ and $y<z$, and nilpotent if there exist $x, y \in(0,1)$ such that $S(x, y)=1$.

Proposition 1. [17, 4] A function $T:[0,1]^{2} \rightarrow[0,1]$ is a continuous Archimedean t-norm iff it has a continuous additive generator, i.e. there exists a continuous strictly decreasing function $t:[0,1] \rightarrow$ $[0, \infty]$ with $t(1)=0$, which is uniquely determined up to a positive multiplicative constant, such that

$$
T(x, y)=t^{-1}(\min (t(x)+t(y), t(0)), \quad x, y \in[0,1]
$$

Proposition 2. [17, 4] A function $S:[0,1]^{2} \rightarrow[0,1]$ is a continuous Archimedean t-conorm iff it has a continuous additive generator, i.e. there exists a continuous strictly increasing function $s:[0,1] \rightarrow$ $[0, \infty]$ with $s(0)=0$, which is uniquely determined up to a positive multiplicative constant, such that

$$
S(x, y)=s^{-1}(\min (s(x)+s(y), s(1)), \quad x, y \in[0,1]
$$

Proposition 3. [13]

A t-norm $T$ is strict if and only if $t(0)=\infty$ holds for each continuous additive generator $t$ of $T$. $A$-norm $T$ is nilpotent if and only if $t(0)<\infty$ holds for each continuous additive generator $t$ of $T$. $A$ t-conorm $S$ is strict if and only if $s(1)=\infty$ holds for each continuous additive generator $s$ of $S$. $A$ t-conorm $S$ is nilpotent if and only if $s(1)<\infty$ holds for each continuous additive generator $s$ of $S$.

In both of the above mentioned Propositions 1 and 2 we can allow the generator functions to be strictly increasing or strictly decreasing, which will result in the fact that they will be determined 
up to a (not necessarily positive) multiplicative constant. For an increasing generator function $t$ of a t-conorm and similarly for a decreasing generator function $s$ of a t-conorm, min in (1) and (2) has to be replaced by max. In this case we will have $t(0)= \pm \infty$ and $s(1)= \pm \infty$ for strict norms and similarly, $t(0)<\infty$ or $t(0)>-\infty$ and $s(1)<\infty$ or $s(1)>-\infty$ for the nilpotent ones.

Proposition 4. [13] Let $T$ be a continuous Archimedean t-norm.

If $T$ is strict, then it is isomorphic to the product $t$-norm $T_{\mathbf{P}}$, i.e., there exists an automorphism of the unit interval $\phi$ such that $T_{\phi}=\phi^{-1}(T(\phi(x), \phi(y)))=T_{\mathbf{P}}$.

If $T$ is nilpotent, then it is isomorphic to the Eukasiewicz $t$-norm $T_{\mathbf{L}}$, i.e., there exists an automorphism of the unit interval $\phi$ such that $T_{\phi}=\phi^{-1}(T(\phi(x), \phi(y)))=T_{\mathbf{L}}$.

From the definitions of t-norms and t-conorms it follows immediately that t-norms are conjunctive, while t-conorms are disjunctive aggregation functions. Therefore, they are widely used as conjunctions and disjunctions in multivalued logical structures.

The logical system based on the nilpotent Łukasiewicz t-norm as conjunction is called Eukasiewiczlogic $[14,18,20]$.

Henceforth we refer to t-norms as conjunctions $(c(x, y))$ and t-conorms as disjunctions $(d(x, y))$.

\subsection{Negations}

Definition 1. A unary operation $n:[0,1] \rightarrow[0,1]$ is called a negation if it is non-increasing and compatible with classical logic, i.e. $n(0)=1$ and $n(1)=0$.

A negation is strict if it is also strictly decreasing and continuous.

A negation is strong, if it is also involutive, i.e. $n(n(x))=x$.

Due to the continuity and strict monotonicity of $n$, for continuous negations there always exists some $\nu_{*}$, for which $n\left(\nu_{*}\right)=\nu_{*}$ holds. $\nu_{*}$ is called the neutral value of the negation and the notation $n_{\nu_{*}}$ stands for a negation operator with neutral value $\nu_{*}$. In the literature $\nu_{*}$ is often denoted by $e$. In Figure 1 we can see some negations with different $\nu_{*}$ values.

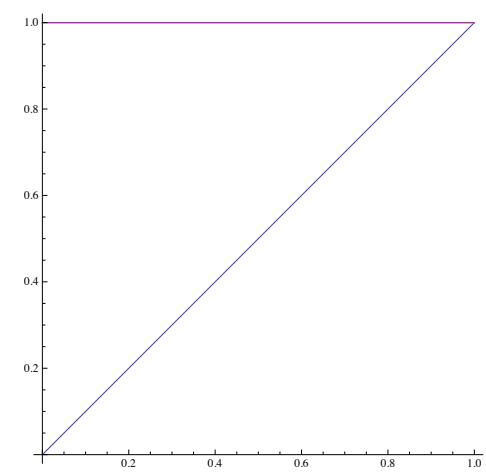

(a) $n_{0}(x)$

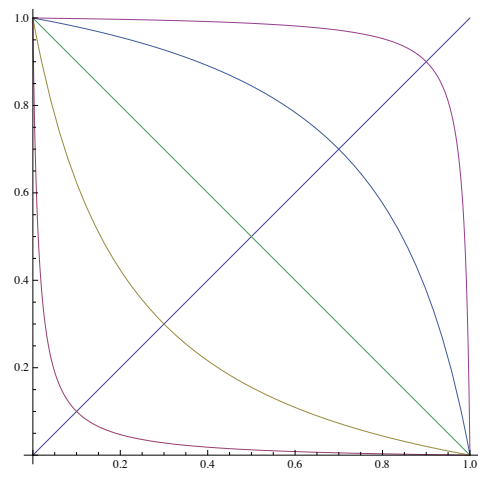

(b) $\nu_{*}=0.1,0.25,0.75,0.9$



(c) $n_{1}(x)$

Figure 1: Continuous negations with different $\nu_{*}$ values and the drastic negations as limit cases

Drastic negations [28] (see Figure 1) are the so-called intuitionistic and dual intuitionistic negations (denoted by $n_{0}$ and $n_{1}$ respectively):

$$
n_{0}(x)=\left\{\begin{array}{lll}
1 & \text { if } & x=0 \\
0 & \text { if } & x>0
\end{array} \text { and } n_{1}(x)=\left\{\begin{array}{lll}
1 & \text { if } & x<1 \\
0 & \text { if } & x=1
\end{array}\right.\right.
$$


These drastic negations are neither continuous nor strictly decreasing, therefore they are not strict negations, but we can get them as limits of negations.

Definition 2. A continuous, strictly increasing function $\varphi:[a, b] \rightarrow[a, b]$ with boundary conditions $\varphi(a)=a, \varphi(b)=b$ is called an automorphism of $[a, b]$.

The well-known representation theorem was obtained by Trillas.

Theorem 1. [25] $n$ is a strong negation if and only if

$$
n(x)=f_{n}(x)^{-1}\left(1-f_{n}(x)\right)
$$

where $f_{n}:[0,1] \rightarrow[0,1]$ is an automorphism of $[0,1]$.

An extension of this result for strict negations was given by Fodor as follows.

Proposition 5. (Lemma 6.2. in [10]) $n$ is a strict negation iff

$$
n(x)=f_{n}^{-1}\left(n^{\prime}\left(f_{n}(x)\right)\right)
$$

where $f_{n}$, called the generator function of $n, f_{n}:[0 ; 1] \rightarrow[0 ; \infty]$ is a strictly monotone, continuous function with $f_{n}(0)=0$ and $f_{n}(1)=1$ and $n^{\prime}$ is a strong negation.

Example 1. For $f_{n}(x)=x^{2}$ and $n^{\prime}(x)=\frac{1-x}{1+x}$ we get $n(x)=\sqrt{\frac{1-x^{2}}{1+x^{2}}}$.

\section{Characterization of strict negation operators}

Our main goal in this section is to present a representation of strict negations with a wide range of examples, since negations will have a very important role to play in the next section.

First let us see some further examples for negation operators. Hamacher proved [15] that the only negation having polynomial form is $1-x$, the so-called standard negation, introduced by Zadeh [29]. He also proved that if an involutive negation belongs to the class of rational polynomials, then it has the following form:

$$
n_{\lambda}(x)=\frac{1-x}{1+\lambda x}, \quad \text { where } \quad \lambda>-1
$$

Sugeno had the same result from the concept of fuzzy measures and integrals [24].

In the literature, generally the standard negation $1-x$ or infrequently $\frac{1-x}{1+x}((4)$ for $\lambda=1)$ are used. Here we make suggestions about using different types of negations as well. The negation operators can be characterized by their neutral values. In [6] (see also [7]) Dombi introduced the following negation formula by expressing $n_{\lambda}(x)$ with the help of its neutral element $\nu_{*}$ :

$$
n_{\nu_{*}}(x)=\frac{1}{1+\left(\frac{1-\nu_{*}}{\nu_{*}}\right)^{2} \frac{x}{1-x}} .
$$

Note that if $\nu_{*} \rightarrow 0$, then $\lim n_{\nu_{*}}(x)=n_{0}(x)$, if $\nu_{*} \rightarrow 1$, then $\lim n_{\nu_{*}}(x)=n_{1}(x)$ and for $\nu_{*}=\frac{1}{2}$ we get the standard negation.

Yager introduced (see [28])

$$
n(x)=\left(1-x^{\alpha}\right)^{1 / \alpha}, \quad \alpha>0 .
$$


Both this type of negation operator and the above-mentioned $n_{\lambda}$ reduce to the standard negation when $\alpha=1$ and $\lambda=0$ respectively.

It is easy to see that the neutral value of the negation operator in (6) is $2^{-\frac{1}{\alpha}}$. If we write this negation operator by using its neutral value as a parameter, we get $n(x)=\left(1-x^{-\frac{1}{\log _{2} \nu_{*}}}\right)^{-\log _{2} \nu_{*}}$.

Note that the representation in Proposition 5 is not unique. It is not always easy to find a generator function. The following propositions state that there can be infinitely many generator functions for a negation operator.

Proposition 6. Let $\nu_{*} \in(0,1), f:[0,1] \rightarrow[0,1]$

$$
f(x)= \begin{cases}\frac{1}{1+\left(\frac{1-\nu_{*}}{\nu_{*}} \cdot \frac{1-x}{x}\right)^{\alpha}} & \text { if } x \neq 0 \\ 0 & \text { if } x=0\end{cases}
$$

is a generator function of the negation $n_{\nu_{*}}($ see (5)) for any $\alpha \neq 0$.

Proof. It can easily be seen that $f^{-1}(x)=\frac{1}{1+\frac{\nu_{*}}{1-\nu_{*}}\left(\frac{1-x}{x}\right)^{\frac{1}{\alpha}}}$, and $1-f(x)=\frac{1}{1+\left(\frac{1-\nu_{*}}{\nu_{*}} \cdot \frac{1-x}{x}\right)^{-\alpha}}$, hence $f^{-1}(1-f(x))=\frac{1}{1+\left(\frac{1-\nu_{*}}{\nu_{*}}\right)^{2} \frac{x}{1-x}}=n_{\nu_{*}}(x)$.

Remark 1. Note that in Proposition 6, if $f$ is a generator function of $n$, then $f^{-1}$ also generates $n$.

Proposition 7. In Theorem 1 (Trillas) the generator function can also be decreasing.

Proof. We shall prove that if $f_{n}$ is a generator function of $n$, then $g_{n}(x)=1-f_{n}(x)$ is also a generator function of $n$. If $f_{n}$ is the generator function of $n$, then $n(x)=f^{-1}\left(1-f_{n}(x)\right)$. If $g_{n}(x)=1-f_{n}(x)$ then $g_{n}^{-1}(x)=f_{n}^{-1}(1-x)$. With this generator function the negation has the following form: $g^{-1}(1-g(x))=$ $=g^{-1}\left(1-\left(1-f_{n}(x)\right)\right)=g^{-1}\left(f_{n}(x)\right)=f_{n}^{-1}\left(1-f_{n}(x)\right)$. Since $f_{n}$ is increasing, $g_{n}$ is decreasing.

For the neutral element $\nu_{*}$, using the representation theorem, we get $\nu_{*}=f^{-1}\left(1-f\left(\nu_{*}\right)\right)$, so $\nu_{*}=f^{-1}\left(\frac{1}{2}\right)$.

For the generator function $g(x)=\frac{a^{x}-1}{a-1}$, where $a>0, a \neq 1$, we get

$$
n(x)=\log _{a}\left(a+1-a^{x}\right) .
$$

If we choose the inverse function $g^{-1}(x)=\log _{a}(x(a-1)+1)$ for the generator function, we obtain

$$
n(x)=\frac{1-x}{1+x(a-1)},
$$

which was mentioned above.

In this section we considered three basic families of strict negations generated by rational, power and exponential functions. (See also Tables 1, 3 and 4.)

\section{Nilpotent connective systems}

Next, instead of operators in themselves, we consider connective systems.

Definition 3. The triple $(c, d, n)$, where $c$ is a t-norm, $d$ is a t-conorm and $n$ is a strong negation, is called a connective system. 
Definition 4. A connective system is nilpotent, if the conjunction $c$ is a nilpotent $t$-norm, and the disjunction $d$ is a nilpotent t-conorm.

Definition 5. Two connective systems $\left(c_{1}, d_{1}, n_{1}\right)$ and $\left(c_{2}, d_{2}, n_{2}\right)$ are isomorphic, if there exists a bijection $\phi:[0,1] \rightarrow[0,1]$ such that

$$
\begin{gathered}
\phi^{-1}\left(c_{1}(\phi(x), \phi(y))\right)=c_{2}(x, y) \\
\phi^{-1}\left(d_{1}(\phi(x), \phi(y))\right)=d_{2}(x, y) \\
\phi^{-1}\left(n_{1}(\phi(x))\right)=n_{2}(x) .
\end{gathered}
$$

In the nilpotent case, the generator functions of the disjunction and the conjunction being determined up to a multiplicative constant can be normalized the following way:

$$
f_{c}(x):=\frac{t(x)}{t(0)}, \quad f_{d}(x):=\frac{s(x)}{s(1)} .
$$

Remark 2. Thus, the normalized generator functions are uniquely defined.

We will use normalized generator functions for conjunctions and disjunctions well. This means that the normalized generator functions of conjunctions, disjunctions and negations are

$$
f_{c}, f_{d}, f_{n}:[0,1] \rightarrow[0,1]
$$

We will suppose that $f_{c}$ is continuous and strictly decreasing, $f_{d}$ is continuous and strictly increasing and $f_{n}$ is continuous and strictly monotone.

Note that by using Proposition 7, there are two special negations generated by the normalized additive generators of the conjunction and the disjunction.

Definition 6. The negations $n_{c}$ and $n_{d}$ generated by $f_{c}$ and $f_{d}$ respectively,

$$
n_{c}(x)=f_{c}^{-1}\left(1-f_{c}(x)\right)
$$

and

$$
n_{d}(x)=f_{d}^{-1}\left(1-f_{d}(x)\right)
$$

are called natural negations.

This means that for a connective system with normalized generator functions $f_{c}, f_{d}$ and $f_{n}$ we can associate three negations by $(3), n_{c}, n_{d}$ and $n$.

Definition 7. Let us define the cutting operation [ ] by

$$
[x]=\left\{\begin{array}{ccc}
0 & \text { if } & x<0 \\
x & \text { if } & 0 \leq x \leq 1 \\
1 & \text { if } & 1<x
\end{array}\right.
$$

and let the notation [ ] also act as 'brackets' when writing the argument of an operator, so that we can write $f[x]$ instead of $f([x])$.

Remark 3. The cutting operator has also been defined in [22]. 
Proposition 8. With the help of the cutting operator, we can write the conjunction and disjunction in the following form, where $f_{c}$ and $f_{d}$ are decreasing and increasing normalized generator functions respectively.

$$
\begin{aligned}
& c(x, y)=f_{c}^{-1}\left[f_{c}(x)+f_{c}(y)\right], \\
& d(x, y)=f_{d}^{-1}\left[f_{d}(x)+f_{d}(y)\right] .
\end{aligned}
$$

Proof. From (1) we know that

$$
c(x, y)=f_{c}^{-1}\left(\min \left(f_{c}(x)+f_{c}(y), f_{c}(0)\right)=f_{c}^{-1}\left(\min \left(f_{c}(x)+f_{c}(y), 1\right)=f_{c}^{-1}\left[f_{c}(x)+f_{c}(y)\right],\right.\right.
$$

and similarly, from $(2)$

$$
d(x, y)=f_{d}^{-1}\left(\min \left(f_{d}(x)+f_{d}(y), f_{d}(0)\right)=f_{d}^{-1}\left(\min \left(f_{d}(x)+f_{d}(y), 1\right)=f_{d}^{-1}\left[f_{d}(x)+f_{d}(y)\right] .\right.\right.
$$

Remark 4. Note that in Proposition 8 it is necessary to use normalized generator functions as the following example shows. This fact supports the use of normalized functions.

Example 2. Let $f_{c}(x)=2-2 x$.

while

$$
c\left(\frac{1}{2}, \frac{1}{2}\right)=f_{c}^{-1}\left(\min \left(f_{c}(x)+f_{c}(y), f_{c}(0)\right)\right)=f_{c}^{-1}(2)=0,
$$

$$
f_{c}^{-1}\left[f_{c}\left(\frac{1}{2}\right)+f_{c}\left(\frac{1}{2}\right)\right]=f_{c}^{-1}[2-1+2-1]=f_{c}^{-1}[2]=f_{c}^{-1}(1)=\frac{1}{2} .
$$

Remark 5. Note that using the cutting function defined above we can omit applying the min and max operators. In the literature, the use of the pseudo-inverse was replaced by the forms (1) and (2), which is now replaced by (9) and (10).

Definition 8. A connective system is called Eukasiewicz system, if it is isomorphic to $([x+y-1],[x+$ $y], 1-x)$, i.e. it has the form $\left(\phi^{-1}[\phi(x)+\phi(y)-1], \phi^{-1}[\phi(x)+\phi(y)], \phi^{-1}[1-\phi(x)]\right) \quad$ for $\forall x, y \in[0,1]$.

Proposition 9. For nilpotent t-norms and t-conorms Definition 6 is equivalent to the following definition (also denoted by $N_{T}$ and $N_{S}$, see [16] (p. 232.) and [2] Definition 2.3.1.):

$$
\begin{array}{ll}
n_{c}(x)=N_{T}(x)=\sup \{y \in[0,1] \mid c(x, y)=0\}, & x \in[0,1], \\
n_{d}(x)=N_{S}(x)=\inf \{y \in[0,1] \mid d(x, y)=1\}, & x \in[0,1] .
\end{array}
$$

Proof. For the conjunction, $c(x, y)=f_{c}^{-1}\left[f_{c}(x)+f_{c}(y)\right]=0$ iff $f_{c}(x)+f_{c}(y) \geq 1$, from which $y \leq$ $f_{c}^{-1}\left(1-f_{c}(x)\right)=n_{c}(x)$. For $y=n_{c}(x), c\left(x, n_{c}(x)\right)=0$ is trivial. The proof is similar for the disjunction as well.

\subsection{Structural properties of connective systems}

Definition 9. Classification property means that the law of contradiction holds, i.e.

$$
c(x, n(x))=0, \quad \forall x, y \in[0,1],
$$

and the excluded third principle holds as well, i.e.

$$
d(x, n(x))=1, \quad \forall x, y \in[0,1] .
$$


Definition 10. The De Morgan identity means that

$$
c(n(x), n(y))=n(d(x, y))
$$

or

$$
d(n(x), n(y))=n(c(x, y)) .
$$

Remark 6. These two forms of the De Morgan law are equivalent, if the negation is involutive. The first De Morgan law holds with a strict negation $n$ if and only if the second holds with $n^{-1}$ (see page 18 in [11])

Definition 11. A connective system is said to be consistent, if the classification property (Definition 9) and the De Morgan identity (Definition 10) hold.

\subsubsection{Classification Property}

Now we will examine the conditions that the connectives and their normalized generator functions in a connective system must satisfy, if we want the classification property to hold.

Proposition 10. (See also [11] 1.5.4. and 1.5.5., and [2] 2.3.2.) In a connective system $(c, d, n)$ the classification property holds iff

$$
n_{d}(x) \leq n(x) \leq n_{c}(x), \quad \text { for } \quad \forall x \in[0,1]
$$

where $n_{c}$ and $n_{d}$ are the natural negations of $c$ and $d$, respectively.

Proof. From the excluded third principle, we have $d(x, n(x))=1$. Using the normalized generator function, $f_{d}^{-1}\left[f_{d}(x)+f_{d}(n(x))\right]=1$. It means that $f_{d}(x)+f_{d}(n(x)) \geq 1$, from which $f_{d}(n(x)) \geq 1-f_{d}(x)$. $f_{d}$ and its inverse $f_{d}^{-1}$ are strictly increasing, thus we get the left hand side of the inequality:

$$
n(x) \geq f_{d}^{-1}\left(1-f_{d}(x)\right)=n_{d}(x) .
$$

Similarly, we get the right hand side from the law of contradiction $c(x, n(x))=0$. Using the normalized generator function we get $f_{c}^{-1}\left[f_{c}(x)+f_{c}(n(x))\right]=0$. From the definition of the cutting function $f_{c}(x)+$ $f_{c}(n(x)) \geq 1$, which means that $f_{c}(n(x)) \geq 1-f_{c}(x)$. Since $f_{c}$ and $f_{c}^{-1}$ are strictly decreasing,

$$
\begin{gathered}
n(x) \leq f_{c}^{-1}\left(1-f_{c}(x)\right)=n_{c}(x), \\
n_{d}(x) \leq n(x) \leq n_{c}(x) .
\end{gathered}
$$

Remark 7. Generally, in a consistent system only one negation is used in the literature. The logical connectives are usually generated by a single generator function.

$$
\begin{gathered}
c(x, y)=f^{-1}[f(x)+f(y)-1], \\
d(x, y)=f^{-1}[f(x)+f(y)], \\
n(x)=f^{-1}(1-f(x)),
\end{gathered}
$$

where $f:[0,1] \rightarrow[0,1]$ is a continuous, strictly increasing function. 
The question arises immediately, whether the use of more than one negation is possible. We will consider this possibility later in detail (see 4.2.1).

Next we give examples for connective systems in which the classification property holds, but which does not fulfil the De Morgan law.

In Section 5, we present an overview of all the examples included in the following part of our paper. The examples from the rational family will be considered in detail in 4.2.1.

Example 3. Let $f_{n}(x):=x^{2}, f_{c}(x):=\sqrt{1-x}$ and $f_{d}(x):=\sqrt{x}$. This connective system fulfills the classification property but does not fulfill the De Morgan law. (See also Table 1.)

We can get another example by using the rational family of normalized generators functions

$$
\begin{array}{ll}
f_{n}(x)=\frac{1}{1+\frac{\nu}{1-\nu} \frac{1-x}{x}}, & f_{n}(0)=0, \\
f_{c}(x)=\frac{1}{1+\frac{\nu_{c}}{1-\nu_{c}} \frac{x}{1-x}}, & f_{c}(1)=0, \\
f_{d}(x)=\frac{1}{1+\frac{\nu_{d}}{1-\nu_{d}} \frac{1-x}{x}}, & f_{d}(0)=0,
\end{array}
$$

choosing e.g. $\nu_{d}=0.3, \nu_{c}=0.7$ and $\nu=0.5$. (See Table 4.)

The existence of such systems explains why we have to consider the De Morgan law in the following section.

\subsubsection{The De Morgan Law}

Now we will examine the conditions that the connectives and their normalized generator functions must satisfy if we want the connective system to fulfill the De Morgan law. Before stating Proposition 12 we need to solve the following functional equation.

Lemma 1. Let $u:[0,1] \longrightarrow[0,1]$ be a continuous, strictly increasing function with $u(0)=0$ and $u(1)=1$. The functional equation

$$
[u(x)+u(y)]=u[x+y]
$$

(where [ ] stands for the cutting operator defined in Definition 7) has a unique solution $u(x)=x$.

Proof. - First we shall prove that $u[0]=0$. Let us suppose that $u[0]=c$, where $0 \leq c \leq 1$. Then

$$
c=u[0+0]=[2 u(0)]
$$

which means $c=[2 c] \quad$ i.e. $\quad c=1$, or $c=0$, but $c=1$ contradicts $u(0)=0$.

- Second, we will show that $u[1]=1$. Similarly, let us suppose that $u[1]=c$, where $0 \leq c \leq 1$. Then $c=u[1+1]=[2 u(1)]$, which means $c=[2 c] \quad$ i.e. $\quad c=1$, or $c=0$, but for $c=0$ we get contradiction.

- Third, we will prove that $u\left(\frac{1}{2}\right)=\frac{1}{2}$.

If $x<\frac{1}{2}$, then $2 x<1 . u$ is strictly increasing, therefore $u(2 x)<1$ as well. $u[2 x]=u(2 x)=$ $2 u(x)=[2 u(x)]$, because of the continuity of $u, \lim _{x \rightarrow \frac{1}{2}} u(2 x)=u(1), 2 \lim _{x \rightarrow \frac{1}{2}} u(x)=1$, which implies $u\left(\frac{1}{2}\right)=\frac{1}{2}$. 
- Similarly, we can prove that $u\left(\frac{1}{2^{m}}\right)=\frac{1}{2^{m}}$.

- Next, we will prove that $u\left(\frac{3}{4}\right)=\frac{3}{4}$.

$u\left(\frac{3}{4}\right)=u\left(\frac{1}{2}+\frac{1}{4}\right)=u\left(\frac{1}{2}\right)+u\left(\frac{1}{4}\right)=\frac{1}{2}+\frac{1}{4}=\frac{3}{4}$.

- In a similar way, we obtain that for $u\left(\frac{k}{2^{m}}\right)=\frac{k}{2^{m}}$.

Then, for any rational number from $[0,1]$, we have $u(x)=x$.

- Let $r$ be any arbitrary irrational number from $[0,1]$. There exists a sequence of rational numbers $q_{n}$ such that $\forall n: q_{n} \in[0,1]$ and $q_{n} \longrightarrow r$.

Because of the continuity of $u$ we have $u\left(q_{n}\right) \longrightarrow u(r)$, which implies $u(r)=r$.

We note that the solution of the following general form of the functional equation (15) can be found in the papers of M. Baczynski [3], [4] (Propositions 3.4. and 3.6.).

Proposition 11. Fix real $a, b>0$. For a function $f:[0, a] \rightarrow[0, b]$, the following statements are equivalent.

1. $f$ satisfies the functional equation

$f(\min (x+y, a))=\min (f(x)+f(y), b) \quad \forall x, y \in[0, a]$.

2. Either $f=b$, or $f=0$, or

$$
f(x)= \begin{cases}0 \text { if } & x=0 \\ b \text { if } & 0<x \leq a\end{cases}
$$

or there exists a unique constant $c \in[b / a, \infty)$ such that

$$
f(x)=\min (c x, b), \quad x \in[0, a] .
$$

Remark 8. Specially, for $a=b=1$ we get the statement of Lemma 1.

Proposition 12. If $f_{c}$ is the normalized generator function of a conjunction in a connective system, $f_{d}$ is a normalized generator function of the disjunction and $n$ is a strong negation, then the following statements are equivalent:

1. The De Morgan law holds in the connective system. That is,

$$
c(n(x), n(y))=n(d(x, y)) .
$$

2. The normalized generator functions of the conjunction, disjunction and negation operator obey the following equations (which are obviously equivalent to each other):

$$
\begin{gathered}
n(x)=f_{c}^{-1}\left(f_{d}(x)\right)=f_{d}^{-1}\left(f_{c}(x)\right) \\
f_{c}(x)=f_{d}(n(x)) \quad \text { or equivalently } \quad f_{d}(x)=f_{c}(n(x)) .
\end{gathered}
$$

Proof. (18) $\Rightarrow(16)$ is obvious.

$(16) \Rightarrow(17)$ : Let us write the De Morgan law using the normalized generator functions.

$$
\left.f_{c}^{-1}\left[f_{c}(n(x))+f_{c}(n(y))\right]\right)=n\left(f_{d}^{-1}\left[f_{d}(x)+f_{d}(y)\right]\right) .
$$


Applying $f_{c}(x)$ to both sides of the equation we obtain

$$
\left[f_{c}(n(x))+f_{c}(n(y))\right]=f_{c}\left(n\left(f_{d}^{-1}\left[f_{d}(x)+f_{d}(y)\right]\right)\right) .
$$

Let us substitute $x=f_{d}^{-1}(x)$. Then we have

$$
\left[f_{c}\left(n\left(f_{d}^{-1}(x)\right)\right)+f_{c}\left(n\left(f_{d}^{-1}(y)\right)\right)\right]=f_{c}\left(n\left(f_{d}^{-1}\left[f_{d}\left(f_{d}^{-1}(x)\right)+f_{d}\left(f_{d}^{-1}(y)\right)\right]\right)\right) .
$$

From this, we get the following functional equation:

$$
\left[f_{c}\left(n\left(f_{d}^{-1}(x)\right)\right)+f_{c}\left(n\left(f_{d}^{-1}(y)\right)\right)\right]=f_{c}\left(n\left(f_{d}^{-1}[x+y]\right)\right) .
$$

If we use $u(x):=f_{c}\left(n\left(f_{d}^{-1}(x)\right)\right)$, then we get the following form of the functional equation:

$$
[u(x)+u(y)]=u[x+y]
$$

We can readily see that function $u(x)$ satisfies the conditions of Lemma 1, i.e. it is a continuous, strictly monotone increasing function with $u(0)=0$ and $u(1)=1$. This means that by Lemma 1 , $u(x)=x$. Hence, $f_{c}\left(n\left(f_{d}^{-1}(x)\right)\right)=x$.

Remark 9. Note that in Proposition 12 any two of $n, f_{c}, f_{d}$ determine the third.

However, note that this remark above does not mean that any two of $n, f_{c}, f_{d}$ can be chosen arbitrary. If $f_{c}$ and $f_{d}$ are given and we want the De Morgan property to hold, we obtain $n$ from (17). This means that for $f_{c}$ and $f_{d}$ the equation in (17) has to hold. Hence, in order to get an involutive negation, we must take notice of the appropriate relationship of the normalized generator functions as the following example shows.

Example 4. Let $f_{c}(x)=1-x^{\alpha}$ and $f_{d}(x)=x^{\beta}$, where $\alpha \neq \beta$. Then

$$
f_{c}^{-1}\left(f_{d}(x)\right)=\sqrt[\alpha]{1-x^{\beta}} \neq \sqrt[\beta]{1-x^{\alpha}}=f_{d}^{-1}\left(f_{c}(x)\right) .
$$

Proposition 13. If the De Morgan property holds in a connective system $(c, d, n)$, then

$$
n_{c}(n(x))=n\left(n_{d}(x)\right)
$$

and similarly,

$$
n_{d}(n(x))=n\left(n_{c}(x)\right),
$$

where $n_{c}$ and $n_{d}$ are the natural negations.

Proof. Because of the involutive property of $n$ it is enough to prove (19).

$$
n\left(f_{c}^{-1}\left(1-f_{c}(n(x))\right)\right)=f_{d}^{-1}\left(f_{c}\left(f_{c}^{-1}\left(1-f_{c}\left(f_{c}^{-1}\left(f_{d}(x)\right)\right)\right)\right)\right)=n_{d}(x) .
$$

Corollary 1. If the De Morgan law holds in a connective system $(c, d, n)$, then

$$
n(x)=n_{c}(x) \text { if and only if } n(x)=n_{d}(x),
$$

where $n_{c}$ and $n_{d}$ are the natural negations. 
Remark 10. Note that we can readily see that if any two of $n, n_{d}, n_{c}$ are equal, then the third is equal to them as well.

Proposition 14. Let $h$ be the transformation for which $h\left(f_{c}(x)\right)=f_{d}(x)$ in a connective system in which the De Morgan property holds. Then $h$ is a (strong) negation.

Proof. By using the involutive property of $n$, we get

$$
\begin{gathered}
f_{d}^{-1}\left(f_{c}(x)\right)=f_{c}^{-1}\left(f_{d}(x)\right), \\
f_{d}(x)=f_{c}\left(f_{d}^{-1}\left(f_{c}(x)\right)\right), \\
f_{c}(x)=f_{d}\left(f_{c}^{-1}\left(f_{d}(x)\right)\right)=h\left(f_{d}(x)\right), \\
f_{c}^{-1}(x)=f_{d}^{-1}\left(h^{-1}(x)\right), \\
f_{d}\left(f_{c}^{-1}(x)\right)=h^{-1}(x)=h(x) .
\end{gathered}
$$

So $h$ is also involutive. It is easy to see that $h(0)=1, h(1)=0$ and $h(x)=f_{d}\left(f_{c}^{-1}(x)\right)$ is strictly monotone decreasing.

Now we give examples for consistent and non-consistent connective systems where the De Morgan property holds. For examples from the rational family of normalized generator functions see propositions 18 and 19.

Example 5. If in a connective system the conjunction, the disjunction and the negation have the following forms

$$
f_{n}(x)=x, f_{c}(x)=(1-x)^{\alpha}, f_{d}(x)=x^{\alpha},
$$

then this connective system is consistent (i.e. the De Morgan law and the classification property hold), if and only if $0<\alpha \leq 1$. (See also Table 1.)

Proof. It is easy to see, that from the Proposition 18 formula (17) is true for the mentioned normalized generator and negation functions:

$$
x^{\alpha}=(1-(1-x))^{\alpha},
$$

which means that the De Morgan law holds.

It is easy to see that the classification property holds if and only if

$$
x^{\alpha}+(1-x)^{\alpha} \geq 1,
$$

which is only true if for $0<\alpha \leq 1$.

Remark 11. Note that the example above shows that there exists a system in which the De Morgan property holds, whereas the classification property does not (for $\alpha>1$ ). (See also Table 1.)

For an example from the rational family of normalized generator functions (see propositions 18 and 19 and also Table 4)

$$
\begin{array}{ll}
f_{n}(x)=\frac{1}{1+\frac{\nu}{1-\nu} \frac{1-x}{x}}, & f_{n}(0)=0, \\
f_{c}(x)=\frac{1}{1+\frac{\nu_{c}}{1-\nu_{c}} \frac{x}{1-x}}, & f_{d}(0)=0, \\
f_{d}(x)=\frac{1}{1+\frac{\nu_{d}}{1-\nu_{d}} \frac{1-x}{x}}, & f_{c}(1)=0,
\end{array}
$$

we can choose e.g. $\nu=0.6, \nu_{c}=0.2$ and $\nu_{d}=0.36$. 
Example 6. If we express the normalized generator functions in Example 5 in terms of the neutral values of the related negations, we get

$$
f_{n}(x)=x, f_{c}(x)=(1-x)^{\frac{1}{\log _{0.5}\left(1-\nu_{c}\right)}}, f_{d}(x)=x^{\log _{\nu_{d}}(0.5)} .
$$

This system fulfills the De Morgan identity iff $\nu_{c}+\nu_{d}=1$, and is consistent iff $\nu_{d} \leq \frac{1}{2}$ also holds. (See also Table 1.)

\subsection{Consistent Connective Systems}

Now we consider the consistent connective systems (in which the De Morgan property and the classification property hold together).

Proposition 15. 1. If the connective system $(c, d, n)$ is consistent, then $f_{c}(x)+f_{d}(x) \geq 1$ for any $x \in[0,1]$, where $f_{c}$ and $f_{d}$ are the normalized generator functions of the conjunction $c$ and the disjunction d respectively.

2. If $f_{c}(x)+f_{d}(x) \geq 1$ for any $x \in[0,1]$ and the De Morgan law holds, then the connective system $(c, d, n)$ satisfies the classification property as well (which now means that the system is consistent).

Proof. By Proposition 10, the classification property holds if and only if

$$
f_{d}^{-1}\left(1-f_{d}(x)\right)=n_{d}(x) \leq n(x) \leq n_{c}(x)=f_{c}^{-1}\left(1-f_{c}(x)\right)
$$

and by Proposition 12, the De Morgan identity holds if and only if

$$
n(x)=f_{d}^{-1}\left(f_{c}(x)\right)=f_{c}^{-1}\left(f_{d}(x)\right) .
$$

From the right hand side of the inequality we get

$$
f_{c}^{-1}\left(f_{d}(x)\right) \leq f_{c}^{-1}\left(1-f_{c}(x)\right)
$$

so

$$
f_{c}(x)+f_{d}(x) \geq 1
$$

Similarly, we get the same from the left hand side of the inequality.

Remark 12. Note that as Example 3 shows, $f_{c}(x)+f_{d}(x) \geq 1$ does not imply the De Morgan law, even if the classification property holds.

Moreover, $f_{c}(x)+f_{d}(x) \geq 1$ without the De Morgan law does not imply the classification property either (for a counterexample we can chose $f_{n}=x^{2}$ and $\alpha=0.7$ in Example 5).

Next, we show examples for consistent systems.

Example 7. If in a connective system the generator function of the conjunction, the disjunction and the negation have the following forms

$$
f_{c}(x)=1-x^{\alpha}, f_{d}(x)=x^{\alpha}, f_{n}(x)=x^{\alpha},
$$

where $\alpha>0$, then the De Morgan law and the classification property hold for every $\alpha$. (See also Table 1.) 
Example 8. More generally, the connective system with generator functions

$$
f_{c}(x)=\left(1-x^{\alpha}\right)^{\frac{\beta}{\alpha}}, f_{d}(x)=x^{\beta}, f_{n}(x)=x^{\alpha},
$$

where $\alpha, \beta>0$ is consistent if and only if $\beta \leq \alpha$. (See also Table 1.)

Note that Example 8 reduces to Example 5 if $\alpha=1$ and $0<\beta \leq 1$ and to Example 7 if $\alpha=\beta$.

Proposition 16. In a connective system the following equations are equivalent:

$$
\begin{gathered}
f_{c}(x)+f_{d}(x)=1 \\
n_{c}(x)=n_{d}(x),
\end{gathered}
$$

where $f_{c}, f_{d}$ are the normalized generator functions of the conjunction and the disjunction and $n_{c}, n_{d}$ are the natural negations.

Proof. From $f_{d}(x)=1-f_{c}(x)$,

$$
f_{d}^{-1}(x)=f_{c}^{-1}(1-x)
$$

and

$$
n_{d}(x)=f_{d}^{-1}\left(1-f_{d}(x)\right)=f_{d}^{-1}\left(1-\left(1-f_{c}(x)\right)\right)=f_{d}^{-1}\left(f_{c}(x)\right)=n(x)=f_{c}^{-1}\left(1-f_{c}(x)\right)=n_{c}(x) .
$$

Remark 13. Let us suppose that in a connective system the De Morgan property holds. If condition (22) holds, then

$$
n_{c}(x)=n(x)=n_{d}(x)
$$

and therefore the system is consistent.

Remark 14. Note that if condition (22) holds, we get the the classical nilpotent (Eukasiewicz) logic.

\subsubsection{Bounded Systems}

The question arises, whether we can use more than one generator functions in our connective system without losing consistency. In the literature only systems generated by only one generator function have been considered, see e.g. [2], Theorem 2.3.18. In these systems the natural negations of the conjunction and the disjunction coincide with the negation operator. Now we will examine the case when $n_{c}(x) \neq n_{d}(x) \neq n(x)$.

Definition 12. A nilpotent connective system is called a bounded system, if

$$
f_{c}(x)+f_{d}(x)>1 \text {, or equivalently } n_{d}(x)<n(x)<n_{c}(x)
$$

holds for all $x \in(0,1)$, where $f_{c}$ and $f_{d}$ are the normalized generator functions of the conjunction and disjunction, and $n_{c}, n_{d}$ are the natural negations.

The following example shows the existence of consistent bounded systems.

Example 9. (See also Table 1.) The connective system generated by

$$
f_{c}(x):=1-x^{\alpha}, f_{d}(x):=1-(1-x)^{\alpha}, n(x):=1-x, \quad \alpha \in(1, \infty]
$$

is a consistent bounded system. 
Proof. Applying (17) from Proposition 12, we obtain: $f_{c}(n(x))=1-(1-x)^{\alpha}=f_{d}(x)$, which means that the De Morgan law holds. It is easy to see that $n_{c}(x)=\sqrt[\alpha]{1-x^{\alpha}}, n_{d}(x)=1-\sqrt[\alpha]{1-(1-x)^{\alpha}}$, i.e.

$$
n_{d}(x)<n(x)<n_{c}(x)
$$

which means that the classification property is also true (see Figure 2).

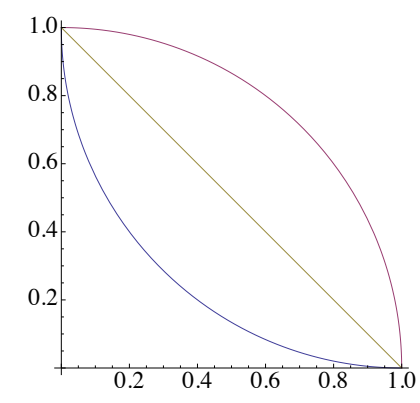

Figure 2: $n_{d}(x)<n(x)<n_{c}(x)$ for $\alpha=2$

For the normalized generator functions we have $f_{c}(x)+f_{d}(x)>1$ for all $x \in(0,1)$.

Remark 15. In Example 9 for $\alpha=1$ we get $n_{d}(x)=n(x)=n_{c}(x)$, i.e. $f_{c}(x)+f_{d}(x)=1$.

Proposition 17. In a connective system $(c, d, n)$, the following statements are equivalent:

$$
\begin{array}{cc}
f_{c}(x)+f_{d}(x)>1 & \text { for all } x \in(0,1), \\
f_{d}\left(f_{c}^{-1}(x)\right)>1-x & \text { for all } x \in(0,1), \\
f_{c}\left(f_{d}^{-1}(x)\right)>1-x & \text { for all } x \in(0,1),
\end{array}
$$

where $f_{c}$ and $f_{d}$ are the normalized generator functions of $c$ and $d$.

Proof. From $n_{d}(x)<n(x)<n_{c}(x)$ we have $f_{d}^{-1}\left(1-f_{d}(x)\right)<f_{c}^{-1}\left(f_{d}(x)\right)$. Substituting $x$ by $f_{d}(x)$ we get $f_{d}^{-1}(1-x)<f_{c}^{-1}(x)$, i.e. $f_{c}\left(f_{d}^{-1}(x)\right)>1-x$, which is also equivalent to $f_{c}\left(f_{d}^{-1}(1-x)\right)>x$.

Next we consider the case of the rational family of the normalized generator functions introduced by Dombi in [6].

Proposition 18. For the Dombi functions (see also Equation (5) and Proposition 6)

$$
\begin{array}{ll}
f_{n}(x)=\frac{1}{1+\frac{\nu}{1-\nu} \frac{1-x}{x}}, & f_{n}(0)=0, \\
f_{c}(x)=\frac{1}{1+\frac{\nu_{c}}{1-\nu_{c}} \frac{x}{1-x}}, & f_{d}(0)=0, \\
f_{d}(x)=\frac{1}{1+\frac{\nu_{d}}{1-\nu_{d}} \frac{1-x}{x}}, & f_{c}(1)=0,
\end{array}
$$

the following statements are equivalent:

1. The connective system generated by the Dombi functions in Proposition 18 satisfies the De Morgan law. 
2. For parameters $\nu_{d}$ and $\nu_{c}$ in the normalized generator functions and for parameter $\nu$ in the negation function the following equation holds:

$$
\left(\frac{1-\nu}{\nu}\right)^{2}=\frac{\nu_{c}}{1-\nu_{c}} \frac{1-\nu_{d}}{\nu_{d}}
$$

Proof. By Proposition 12, the De Morgan law holds iff:

$$
f_{c}(n(x))=f_{d}(x) .
$$

From Proposition 6 for $\alpha=-1$ we know that

$$
n(x)=\frac{1}{1+\left(\frac{1-\nu}{\nu}\right)^{2} \frac{x}{1-x}},
$$

so

$$
f_{c}(n(x))=\frac{1}{1+\left(\frac{\nu_{c}}{1-\nu_{c}}\right)\left(\frac{\nu}{1-\nu}\right)^{2} \frac{1-x}{x}}=\frac{1}{1+\frac{\nu_{d}}{1-\nu_{d}} \frac{1-x}{x}} .
$$

This means that the equality (28) holds if and only if the parameters on the left and the right hand side are equal, i.e.:

$$
\left(\frac{1-\nu}{\nu}\right)^{2}=\frac{\nu_{c}}{1-\nu_{c}} \frac{1-\nu_{d}}{\nu_{d}}
$$

Remark 16. From (30) we get that the De Morgan law holds iff

$$
\nu=\frac{1}{1+\sqrt{\frac{\nu_{c}}{1-\nu_{c}} \frac{1-\nu_{d}}{\nu_{d}}}} .
$$

Proposition 19. For the natural negations derived from the Dombi functions defined in Proposition 18, the following statements are equivalent for $x \in(0,1)$ :

$$
\begin{gathered}
n_{d}(x)<n(x)<n_{c}(x), \\
\nu_{d}<\nu<\nu_{c} .
\end{gathered}
$$

Proof.

$$
\frac{1}{1+\left(\frac{1-\nu_{d}}{\nu_{d}}\right)^{2} \frac{x}{1-x}}<\frac{1}{1+\left(\frac{1-\nu}{\nu}\right)^{2} \frac{x}{1-x}}
$$

(see Table 5) if and only if $\nu_{d}<\nu$. Similarly, we can prove the other side of the inequality as well.

Remark 17. Note that if the De Morgan property holds,

$$
f_{c}(x)+f_{d}(x)>1
$$

is also equivalent to (32) and (33).

Proposition 20. For the Dombi functions defined in Proposition 18, the followings are equivalent for $x \in(0,1)$ :

$$
\begin{gathered}
f_{c}(x)+f_{d}(x)>1, \\
\nu_{c}+\nu_{d}<1 .
\end{gathered}
$$


Figure 3: The relationship between $\nu, \nu_{c}$ and $\nu_{d}$ in consistent rational systems

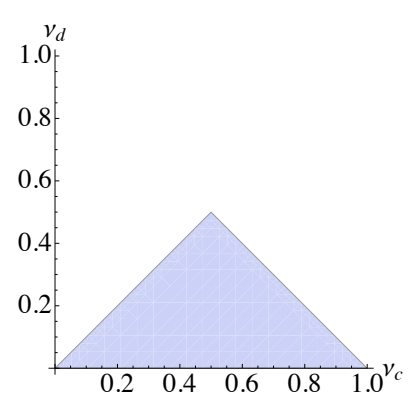

(a) The relationship between $\nu_{c}$ and $\nu_{d}$

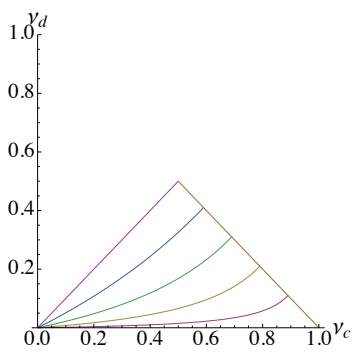

(b) The relationship of $\nu_{c}$ and $\nu_{d}$ for different fixed values of $\nu$



(c) $\nu$ as a function of $\nu_{c}$ and $\nu_{d}$

Proof.

if and only if

$$
\frac{1}{1+\left(\frac{\nu_{c}}{1-\nu_{c}} \frac{x}{1-x}\right)}>1-\frac{1}{1+\left(\frac{\nu_{d}}{1-\nu_{d}} \frac{1-x}{x}\right)}=\frac{1}{1+\left(\frac{1-\nu_{d}}{\nu_{d}} \frac{x}{1-x}\right)}
$$

$$
\frac{\nu_{c}}{1-\nu_{c}}<\frac{\nu_{d}}{1-\nu_{d}}
$$

which is equivalent to $\nu_{c}+\nu_{d}<1$.

Remark 18. Note that if the De Morgan property holds,

$$
n_{d}(x)<n(x)<n_{c}(x)
$$

is also equivalent to (35) and (36).

The relationship between $\nu_{c}$ and $\nu_{d}$ from Propositions 19 and 20 can be seen in Figure 3a. In Figure 3b we can see the possible values of $\nu_{c}$ and $\nu_{d}$ for fixed values of $\nu$. The values of $\nu$ as a function of $\nu_{c}$ and $\nu_{d}$ can be seen on Figure 3c.

Remark 19. By using (37), (36) and (31) we obtain that in a consistent system with $f_{c}(x)+f_{d}(x)>1, \nu<\frac{1}{2}$ always holds.

Remark 20. For $\nu=\frac{1}{2}$ we get $\sqrt{\frac{\nu_{c}}{1-\nu_{c}} \frac{1-\nu_{d}}{\nu_{d}}}=1$, so $\nu_{c}=\nu_{d}=\nu=\frac{1}{2}$.

Example 10. For $\nu_{c}=0.5$ and $\nu_{d}=0.1 \nu=0.25, \nu_{c}+\nu_{d}<1$ and $n_{d}(x)<n(x)<n_{c}(x)$.

See Figure 4.

In Figure 5a and 5b examples for conjunctions and disjunctions are shown for $f_{c}(x)+f_{d}(x)=1$ and for $f_{c}(x)+f_{d}(x)>1$ respectively. Note that the coincidence and the separation of $n_{c}$ and $n_{d}$ (see their alternative definition in Proposition 9 as well) can easily be seen. 
Figure 4: Normalized generators of a consistent system (Example 10)



(a) $f_{n}(x)$ for $\nu=0.25$

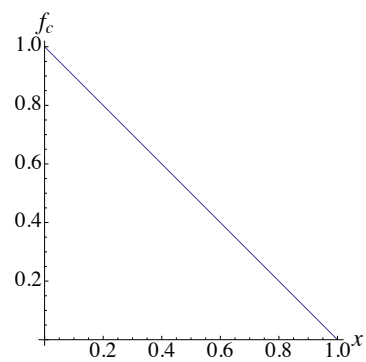

(b) $f_{c}(x)$ for $\nu_{c}=0.5$



(c) $f_{d}(x)$ for $\nu_{d}=0.1$

Figure 5: Conjunction $c[x, y]$ and disjunction $d[x, y]$



(a) $\nu_{c}=0.6$ and $\nu_{d}=0.4\left(\nu_{c}+\nu_{d}=1\right)$

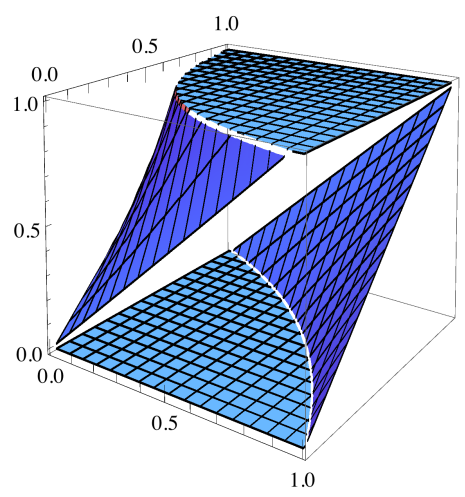

(b) $\nu_{c}=0.4$ and $\nu_{d}=0.3\left(\nu_{c}+\nu_{d}<1\right)$

\section{Overview of examples}

In this section we give an overview of the three families of normalized generator functions used in our examples and propositions, namely power, exponential and rational functions (see also (5), (6) and (7).) For the power and the rational normalized generator functions the logical connectives are also given. In the case of the rational and in a special case of the power functions we give the normalized generators in terms of the neutral values as well. Finally, we give some examples of consistent connective systems with mixed types of normalized generator functions. 


\begin{tabular}{|c|c|c|c|c|c|c|}
\hline & $f_{n}$ & $f_{c}$ & $f_{d}$ & Classification & De Morgan & Remarks \\
\hline Example 3 & $x^{2}$ & $\sqrt{\sqrt{1-x}}$ & $\sqrt{x}$ & $\bar{~} \bar{\checkmark}$ & - & \\
\hline Example 5 & $x$ & $(1-x)^{\alpha}$ & $x^{\alpha}$ & $\checkmark$ & $\checkmark$ & $0<\alpha \leq 1$ \\
\hline Remark 11 & $x$ & $(1-x)^{\alpha}$ & $x^{\alpha}$ & - & $\checkmark$ & $\alpha>1$ \\
\hline Example 6 & $x$ & $(1-x)^{\frac{1}{\log _{0.5}\left(1-\nu_{c}\right)}}$ & $x^{\log _{\nu_{d}} 0.5}$ & iff $\nu_{d} \leq 0.5$ & iff $\nu_{c}+\nu_{d}=1$ & $\begin{array}{l}\text { Example } 5 \text { and Re- } \\
\text { mark } 11 \text { in terms of } \\
\text { the neutral value }\end{array}$ \\
\hline Example 7 & $x^{\alpha}$ & $1-x^{\alpha}$ & $x^{\alpha}$ & $\checkmark$ & $\checkmark$ & $\alpha>0$ \\
\hline Example 8 & $x^{\alpha}$ & $\left(1-x^{\alpha}\right)^{\frac{\beta}{\alpha}}$ & $x^{\beta}$ & $\checkmark$ & $\checkmark$ & $\beta \leq \alpha ; \quad \alpha, \beta>0$ \\
\hline Example 9 & $x$ & $1-x^{\alpha}$ & $1-(1-x)^{\alpha}$ & $\checkmark$ & $\checkmark$ & $\begin{array}{l}\alpha \quad \geq \\
f_{c}+f_{d}>1 \text { iff } \\
\alpha>1\end{array}$ \\
\hline
\end{tabular}

Table 1: Power functions as normalized generators

\begin{tabular}{|l|l|l|l|c|c|l|}
\hline & $f_{n}$ & $f_{c}$ & $f_{d}$ & $n(x)$ & $c(x, y)$ & $d(x, y)$ \\
\hline \hline E 3 & $x^{2}$ & $\sqrt{1-x}$ & $\sqrt{x}$ & $\sqrt{1-x^{2}}$ & $1-[\sqrt{(1-x)}+\sqrt{(1-y)}]^{2}$ & {$[\sqrt{x}+\sqrt{y}]^{2}$} \\
\hline E 5 & $x$ & $(1-x)^{\alpha}$ & $x^{\alpha}$ & $1-x$ & $1-\left[(1-x)^{\alpha}+(1-y)^{\alpha}\right]^{\frac{1}{\alpha}}$ & {$\left[x^{\alpha}+y^{\alpha}\right]^{\frac{1}{\alpha}}$} \\
\hline R 11 & $x$ & $(1-x)^{\alpha}$ & $x^{\alpha}$ & $1-x$ & $1-\left[(1-x)^{\alpha}+(1-y)^{\alpha}\right]^{\frac{1}{\alpha}}$ & {$\left[x^{\alpha}+y^{\alpha}\right]^{\frac{1}{\alpha}}$} \\
\hline E 7 & $x^{\alpha}$ & $1-x^{\alpha}$ & $x^{\alpha}$ & $\sqrt[\alpha]{1-x^{\alpha}}$ & $\left(1-\left[2-x^{\alpha}-y^{\alpha}\right]\right)^{\frac{1}{\alpha}}$ & {$\left[x^{\alpha}+y^{\alpha}\right]^{\frac{1}{\alpha}}$} \\
\hline E 8 & $x^{\alpha}$ & $\left(1-x^{\alpha}\right)^{\frac{\beta}{\alpha}}$ & $x^{\beta}$ & $\sqrt[\alpha]{1-x^{\alpha}}$ & $\left(1-\left[\left(1-x^{\alpha}\right)^{\frac{\beta}{\alpha}}+\left(1-y^{\alpha}\right)^{\frac{\beta}{\alpha}}\right]^{\frac{\alpha}{\beta}}\right)^{\frac{1}{\alpha}}$ & {$\left[x^{\beta}+y^{\beta}\right]^{\frac{1}{\beta}}$} \\
\hline E 9 & $x$ & $1-x^{\alpha}$ & $1-(1-x)^{\alpha}$ & $1-x$ & $\left(1-\left[2-x^{\alpha}-y^{\alpha}\right]\right)^{\frac{1}{\alpha}}$ & 1 \\
\end{tabular}

Table 2: Power functions as normalized generators - logical connectives

\begin{tabular}{|l|l|l|l|c|l|}
\hline & $f_{n}$ & $f_{c}$ & $f_{d}$ & De Morgan law & Consistency \\
\hline \hline & $\frac{a^{x}-1}{a-1}$ & $\frac{\left(a+1-a^{x}\right)^{\log _{a^{b}}-1}}{b-1}$ & $\frac{b^{x}-1}{b-1}$ & $\checkmark$ & $\begin{array}{l}\text { Consistent for e.g. } \\
a=0.5, b=0.7 \text { or } \\
\end{array}$ \\
& & & & \\
& & & & \\
& & & & \\
\end{tabular}

Table 3: Exponential functions as normalized generators 


\begin{tabular}{|c|c|c|c|c|c|}
\hline & $f_{n}$ & $f_{c}$ & $f_{d}$ & Classification & De Morgan \\
\hline $\begin{array}{l}\text { Propositions } \\
18 \text { and } 19\end{array}$ & $\frac{1}{1+\frac{\nu}{1-\nu} \frac{1-x}{x}}$ & $\frac{1}{1+\frac{\nu_{c}}{1-\nu_{c}} \frac{x}{1-x}}$ & $\frac{1}{1+\frac{\nu_{d}}{1-\nu_{d}} \frac{1-x}{x}}$ & $\nu_{d}<\nu<\nu_{c}$ & $\begin{array}{l}\left(\frac{1-\nu}{\nu}\right)^{2}=\frac{\nu_{c}}{1-\nu_{c}} \frac{1-\nu_{d}}{\nu_{d}} \\
\nu=\frac{1}{1+\sqrt{\frac{\nu_{c}}{1-\nu_{c}}} \frac{1-\nu_{d}}{\nu_{d}}}\end{array}$ \\
\hline Example 3 & $\nu=0.5$ & $\nu_{c}=0.7$ & $\nu_{d}=0.3$ & $\checkmark$ & - \\
\hline Remark 11 & $\nu=0.6$ & $\nu_{c}=0.2$ & $\nu_{d}=0.36$ & - & $\checkmark$ \\
\hline Example 10 & $\nu=0.25$ & $\nu_{c}=0.5$ & $\nu_{d}=0.1$ & $\checkmark$ & $\checkmark$ \\
\hline
\end{tabular}

Table 4: Rational functions as normalized generators

\begin{tabular}{|l|l|l|l|l|}
\hline & $f(x)$ (normalized generator) & $f^{-1}(x)$ & $1-f(x)$ & negation \\
\hline \hline negation & $\frac{1}{1+\frac{\nu}{1-\nu} \frac{1-x}{x}}$ & $\frac{1}{1+\frac{1-\nu}{\nu} \frac{1-x}{x}}$ & $\frac{1}{1+\frac{1-\nu}{\nu} \frac{x}{1-x}}$ & $n(x)=\frac{1}{1+\left(\frac{1-\nu}{\nu}\right)^{2} \frac{x}{1-x}}$ \\
\hline conjunction & $\frac{1}{1+\frac{\nu_{c}}{1-\nu_{c}} \frac{x}{1-x}}$ & $\frac{1}{1+\frac{1-\nu_{c}}{\nu_{c}} \frac{x}{1-x}}$ & $\frac{1}{1+\frac{1-\nu_{c}}{\nu_{c}} \frac{1-x}{x}}$ & $n_{c}(x)=\frac{1}{1+\left(\frac{\nu_{c}}{1-\nu_{c}}\right)^{2} \frac{x}{1-x}}$ \\
\hline disjunction & $\frac{1}{1+\frac{\nu_{d}}{1-\nu_{d}} \frac{1-x}{x}}$ & $\frac{1}{1+\frac{1-\nu_{d}}{\nu_{d}} \frac{1-x}{x}}$ & $\frac{1}{1+\frac{1-\nu_{d}}{\nu_{d}} \frac{x}{1-x}}$ & $n_{d}(x)=\frac{1}{1+\left(\frac{1-\nu_{d}}{\nu_{d}}\right)^{2} \frac{x}{1-x}}$ \\
\hline
\end{tabular}

Table 5: Rational functions as normalized generators -3 negations

\begin{tabular}{|l|l|l|l|c|l|}
\hline & $f_{n}$ & $f_{c}$ & $f_{d}$ & De Morgan law & Consistency \\
\hline \hline $\begin{array}{l}\text { Rational and } \\
\text { power }\end{array}$ & $\frac{1}{1+\frac{\nu}{1-\nu} \frac{1-x}{x}}$ & $\left(\frac{1}{1+\left(\frac{1-\nu}{\nu}\right)^{2} \frac{x}{1-x}}\right)^{\alpha}$ & $x^{\alpha}$ & $\checkmark$ & $\begin{array}{l}\text { Consistent for e.g. } \\
\alpha=1, \nu=0.8 \text { or } \\
\alpha=2, \nu=0.9\end{array}$ \\
\hline $\begin{array}{l}\text { Power and } \\
\text { exponential }\end{array}$ & $x^{\alpha}$ & $\frac{a^{\left(1-x^{\alpha}\right)^{\frac{1}{\alpha}}-1}}{a-1}$ & $\frac{a^{x}-1}{a-1}$ & $\checkmark$ & $\begin{array}{l}a>0, a \neq 1, \alpha>0 . \\
\text { Consistent for e.g. } \\
\alpha=1, a=0.5\end{array}$ \\
\hline
\end{tabular}

Table 6: Mixed types of normalized generator functions

\section{Conclusion}

After giving a characterization and a wide range of examples for negation operators, we have studied connective systems in which the conjunction, the disjunction and the negation are generated by bounded and normalized functions. Three negations can be naturally associated with the normalized generator functions, $n_{c}, n_{d}$ and $n$. Necessary and sufficient conditions of the classification property (the excluded middle and the law of contradiction), the De Morgan law and consistency have been given. We thoroughly examined the question whether the three negations can differ from one another in a con- 
sistent system. The positive answer means that a consistent system generated by nilpotent operators is not necessarily isomorphic to Łukasiewicz logic. We get a system isomorphic to Łukasiewicz logic if and only if the three negations coincide. Finally, we have also given several examples for consistent systems with three different negations.

\section{Acknowledgments}

The authors are grateful to all anonymous referees whose comments and suggestions have significantly improved our original version of this paper. This work was partially supported by AÖU's $870 ̈ u 3$ project and partially supported by the European Union and the European Social Fund through project FuturICT.hu (grant no.: TÁMOP-4.2.2.C-11/1/KONV-2012-0013). The authors gratefully acknowledge the financial support by the Óbuda University Research Fund. 


\section{References}

[1] J. Aczél, Über eine Klasse von Funktional-gleichungen, Commentarii mathematici Helvetici, Vol. 21, p. 247-252, 1948.

[2] M. Baczyński and B. Jayaram, Fuzzy Implications, Springer-Verlag Berlin Heidelberg, 2008.

[3] M. Baczyński and B. Jayaram, On the Distributivity of Fuzzy Implications Over Nilpotent or Strict Triangular Conorms, IEEE Transactions on Fuyyz Systems, Vol. 17, No. 3, p. 590-603, 2009.

[4] M. Baczyński, On the distributivity of fuzzy implications over continuous and Archimedean triangular conorms, Fuzzy Sets and Systems 161, p. 1406-1419, 2010.

[5] G. Beliakov, A. Pradera, T. Calvo, Aggregation Functions: A Guide for Practitioners, SpringerVerlag, Berlin, Heidelberg, 2007.

[6] J. Dombi, Towards a General Class of Operators for Fuzzy Systems, IEEE Transaction on Fuzzy Systems, Vol. 16, 477-484, 2008.

[7] J. Dombi, One class of continuous valued logical operators and its applications: Pliant concept, 2009.

[8] D. Dubois and H. Prade, Fuzzy sets in approximate reasoning. Part 1: Inference with possibility distributions, Fuzzy Sets and Systems Vol. 40, p. 143-202, 1991.

[9] F. Esteva, E. Trillas and X. Domingo, Weak and strong negation function for fuzzy set theory, In Eleventh IEEE International Symposium on Multi-Valued Logic, p. 23-27, Norman, Oklahoma, 1981.

[10] J.C. Fodor, A new look at fuzzy connectives, Fuzzy Sets and Systems, Vol. 57, p. 141-14857, 1993.

[11] J.C. Fodor, M. Roubens, Fuzzy Preference Modelling and Multicriteria Decision Support, Kluwer, Dordrecht, 1994.

[12] S. Gottwald, A Treatise on Many-Valued Logics. Studies in Logic and Computation, Vol. 9, Research Studies Press, Baldock, Hertfordshire, England, 2001.

[13] M. Grabish, J. Marichal, R. Mesiar and E. Pap, Aggregation Functions, Cambridge University Press, New York, 2009.

[14] P. Hájek, Metamathematics of Fuzzy Logic, Kluwer Academic Publishers, Dordrecht, 1998.

[15] H. Hamacher, Über Logische Aggregationen Nicht-Binar Explizierter Entscheidungskriterien, Rit G Fischer Verlag, 1978.

[16] E. P. Klement, R. Mesiar, E. Pap, Triangular Norms, Kluwer Academic Publishers, Boston, London, Dordrecht, 2000.

[17] C. Ling, Representation of associative functions, Publ. Math. Debrecen, Vol. 12, 189-212, 1965.

[18] J. Łukasiewicz, On three-valued logic, in L. Borkowski (ed.), Selected works by Jan Łukasiewicz, North-Holland, Amsterdam, 1970, p. 87-88. 
[19] V. Novák, I. Perfilieva, J. Močkor, Mathematical Principles of Fuzzy Logic. Kluwer Academic publishers, Boston, Dordrecht, London, 1999.

[20] H. Ono, Substructural logics and residuated lattices - an introduction, in F.V. Hendricks, J. Malinowski (eds.): Trends in Logic: 50 Years of Studia Logica, Trends in Logic, Vol. 20, p. 177-212, 2003.

[21] R. Rothenberg, Łukasiewicz's many valued logic as a Doxastic Modal Logic, Dissertation, University of St. Andrews, 2005.

[22] M. Sabo and P. Strezo, On Reverses of Some Binary Operations, Kybernetika, Vol. 41, p. 425-434, 2005.

[23] B. Schweizer and A. Sklar, Probabilistic Metric Spaces, North-Holland, Amsterdam, 1983.

[24] M. Sugeno, Fuzzy Measures and Fuzzy Integrals, North Holland, 1997.

[25] E. Trillas, Sorbe funciones de negación en la teoría de conjuntos difusos, Stochastica, Vol. III., p. 47-60, 1979.

[26] E. Trillas and L. Valverde, On some functionally expressible implications for fuzzy set theory, Proc. of the 3rd International Seminar on Fuzzy Set Theory, Linz, Austria, p. 173-190, 1981.

[27] S. Weber, A general concept of fuzzy connectives, negations and implications based on t-norms and t-conorms, Fuzzy Sets and Systems, Vol. 11, p. 115-134, 1983.

[28] R. R. Yager, On the measure of fuzziness and negation, Information and Control, Vol. 44, p. 236-260, 1980.

[29] L. A. Zadeh, Fuzzy Sets, Information and Control, Vol. 8, p. 338-353, 1965. 